\title{
OTIMIZAÇÃO DE CONDIÇÕES DE CULTIVO HETEROTRÓFICO DE MICROALGAS
}

\author{
J. B. da SILVEIRA ${ }^{1}$; J. P. LORENSI ${ }^{1}$; R. S. COELHO ${ }^{1} ;$ T. T. FRANCO ${ }^{1}$ \\ ${ }^{1}$ Universidade Estadual de Campinas, Faculdade de Engenharia Química \\ Email para contato: franco.feq@gmail.com
}

\begin{abstract}
RESUMO - Devido à crescente preocupação com os impactos ambientais causados pelo uso de combustíveis fósseis e o incentivo para utilização de fontes de energia limpas e renováveis, a produção de biocombustíveis a partir de microalgas tem sido bastante explorada recentemente, principalmente devido às altas taxas de crescimento e capacidade de acúmulo de lipídeos que as microalgas apresentam. Com o objetivo de investigar o efeito da razão $\mathrm{C} / \mathrm{N}$ sobre o crescimento heterotrófico da microalga Chlorella vulgaris, o presente trabalho apresenta resultados de ensaios em três diferentes valores de razão carbono nitrogênio (20, 40 e $60 \mathrm{~g} \mathrm{C} / \mathrm{g} \mathrm{N})$. O crescimento da biomassa, a concentração de glicose e nitrato e o acúmulo de lipídeos foram avaliados durante todo o processo. Os resultados finais indicam que a maior concentração de biomassa máxima ocorreu para a relação $\mathrm{C} / \mathrm{N} 40(4,71 \pm 0,09 \mathrm{~g} / \mathrm{L})$, enquanto que a maior porcentagem de lipídeos se deu para a relação $\mathrm{C} / \mathrm{N} 60(33,58 \pm 0,43 \%)$. A produtividade máxima de biomassa, por sua vez, também foi maior para a relação $\mathrm{C} / \mathrm{N} 40(0,043 \pm 0,001 \mathrm{~g} / \mathrm{L} \mathrm{h})$.
\end{abstract}

\section{INTRODUÇÃO}

Devido à crescente preocupação com os impactos ambientais causados pelo uso de combustíveis fósseis e o incentivo para utilização de fontes de energia limpas e renováveis, os combustíveis oriundos da biomassa ganham importância. Em meio a este cenário, pesquisas e estudos vêm sendo conduzidos a fim de consolidar as microalgas como uma fonte de matériaprima viável e promissora para a produção de biocombustíveis, tais como: biodiesel, derivado do óleo das microalgas; bio-metano, derivado da biomassa; bio-hidrogênio; bio-óleo e bioetanol, derivado de açúcares e amido (Franco et al., 2013, Rashid et al., 2014); (Gouveia, 2011). O biodiesel é um biocombustível interessante, pois é renovável, biodegradável e não tóxico (Giordano et al., 2014).

Biocombustíveis de primeira geração são tradicionalmente produzidos a partir de oleaginosas e gorduras animais, biocombustíveis de segunda e terceira geração são derivados de matérias-primas não alimentares, como resíduos (resíduos da indústria de processamento de madeira, cascas de frutas, entre outros) no caso daqueles de segunda geração, e as microalgas, para terceira geração (Kiran et al.). As microalgas são organismos clorofilados encontrados tanto em ambientes terrestres úmidos quanto em ambientes aquáticos. Podem ser cultivadas de modo autotrófico (obtém energia da luz e a fonte de carbono é o $\mathrm{CO}_{2}$ do ar e, através da fotossíntese, sintetizam suas biomoléculas), heterotrófico (compostos orgânicos são utilizados como fontes de energia e de carbono), mixotrófico ou foto-heterotrófico. Embora a 
produtividade em biomassa e em lipídios dependa da cepa utilizada, o metabolismo heterotrófico pode ser mais produtivo do que outras condições de cultivo (Franco et al., 2013); tal fato pode ser explicado, por exemplo, pelo suprimento de luz, pois, à medida que a cultura se torna mais densa, a penetração de luz cai drasticamente, reduzindo a produtividade em biomassa e o conteúdo lipídico (Giordano et al., 2014). Certas espécies de algas podem crescer exclusivamente em substratos orgânicos e isto se tornou uma opção interessante para a produção de biocompostos. As microalgas ganharam tanto destaque como uma matéria-prima em potencial para a produção de biocombustíveis porque, dependendo da espécie e das condições de cultivo, podem acumular quantidades consideráveis de polissacarídeos e triglicerídeos (Slade and Bauen, 2013). Sistemas heterotróficos podem aumentar muito a produtividade em biomassa e lipídeos, o que é importante para diminuir o custo de produção quando comparado aos sistemas autotróficos. Dentre as microalgas apropriadas para a heterotrofia, a Chlorella foi muito estudada e mostrou um alto potencial para a produção de lipídeos: em certas espécies de Chlorella o conteúdo lipídico pode variar de 10 a 55\% do peso seco. A composição do meio de cultivo, principalmente as fontes de carbono e nitrogênio, é um fator crítico para o crescimento das microalgas. Assim, para obter maior acúmulo de biomassa e lipídeos é importante encontrar as fontes apropriadas de carbono e nitrogênio e sua concentração ótima (Isleten - Hosoglu et al., 2012).

As microalgas apresentam algumas vantagens quando comparadas aos vegetais superiores, tais como: maior eficiência energética; crescimento acelerado; não competição com alimentos quanto ao uso de terras agricultáveis, pois podem ser cultivadas em áreas marginais; não competição por uso de água fresca, pois podem utilizar água proveniente de reuso; biorremediação de águas residuais através da remoção de $\mathrm{NH}_{4}{ }^{+}, \mathrm{NO}_{3}, \mathrm{PO}_{4}{ }^{3-}$ e metais pesados; alta capacidade de sequestro de $\mathrm{CO}_{2}$; produção não sazonal, o que possibilita sua utilização o ano todo; possibilidade de induzir maior produção do produto desejado; capacidade de crescer sob condições ambientais extremas e pouca oferta de nutrientes, e impacto muito menor sobre o meio ambiente do que as culturas produtoras convencionais (Franco et al., 2013, Gouveia, 2011, Kiran et al.). Alguns números e dados são capazes de quantificar e comprovar essas vantagens: as algas podem crescer de 20 a 30 vezes mais rápido do que as culturas alimentares e tem um ciclo de colheita de 1-10 dias, o que permite várias colheitas num período de tempo muito curto; o rendimento de óleo de certas variedades de algas pode ser 300 vezes maior que o do milho, 130 vezes maior do que a partir da soja, 30 vezes maior do que a partir de pinhão, e cerca de 10 vezes maior do que o óleo de palma, por hectare de terra em uma base anual. Por estas razões, os combustíveis à base de algas são a opção mais eficaz e sustentável para as alterações climáticas e a única fonte de energia renovável que tem capacidade para atender a demanda mundial por combustíveis no longo prazo (Ziolkowska and Simon, 2014). São todos esses fatores que fazem com que pesquisas e estudos sejam conduzidos no mundo todo a fim de desenvolver a tecnologia necessária para tornar a produção de lipídeos de algas um processo industrial (Wijffels and Barbosa, 2010).

O biodiesel de algas é produzido pela transesterificação do óleo, portanto, o rendimento da produção depende da quantidade de óleo nas células das microalgas. Sendo assim, para tornar economicamente viável esta produção, é preciso otimizar o meio de cultivo a fim de que as algas cresçam mais e ao mesmo tempo acumulem mais lipídeos (Franco et al., 2013).

O carbono é o principal componente da biomassa das algas, representando cerca de $50 \%$ do peso seco. Em heterotrofia, os substratos orgânicos mais comumente utilizados são os açúcares, especialmente glicose, e podem ser usados para aumentar a produção tanto de 
biomassa quanto de lipídeos. A concentração de nitrogênio também é um fator importante para o metabolismo de lipídeos. O nitrato foi sugerido como mais adequado quando comparado com outras fontes de nitrogênio, como ureia e amônia, para a produção de lipídeos. Além do carbono e nitrogênio, limitações nas quantidades de fósforo e enxofre, por exemplo, são também capazes de influenciar o acúmulo de lipídeos. (Liu et al., 2011).

Este trabalho tem como objetivo estudar a modificação da relação carbono e nitrogênio de um cultivo em heterotrofia, utilizando glicose como fonte de carbono, e como essa mudança influencia na concentração de biomassa, no percentual de lipídios e na produtividade de biomassa da microalga Chlorella vulgaris CPCC 90.

\section{MATERIAIS E MÉTODOS}

\subsection{Microorganismo e Preparo do inóculo}

O microorganismo utilizado no estudo foi Chlorella vulgaris CPCC 90. Para o preparo do inóculo, células foram removidas de uma cultura em ágar inclinado através da adição de meio de cultivo BBM e remoção mecânica com o auxílio de uma alça de inoculação estéril. A suspensão de células resultante foi adicionada a frascos tipo Erlenmeyer contendo caldo BBM. Os frascos foram incubados em shaker por 3 dias a $140 \mathrm{RPM}$ a $26{ }^{\circ} \mathrm{C}$.

\subsection{Cultivo}

O meio de cultura utilizado foi o meio sintético Bold's Basal Medium (BBM), cuja composição pode ser obsevada na Tabela 1, e no qual as algas comprovadamente apresentam bom crescimento.

Tabela 1 - Composição do meio sintético Bold's Basal Medium (BBM)

\begin{tabular}{llll}
\hline Composto & Concentração (g/L) & Composto & Concentração (g/L) \\
\hline $\mathrm{KH}_{2} \mathrm{PO}_{4}$ & 0,175 & $\mathrm{NaCl}$ & 0,025 \\
$\mathrm{CaCl}_{2} \cdot 2 \mathrm{H}_{2} \mathrm{O}$ & 0,025 & $\mathrm{Na}_{2}$ EDTA KOH & 0,1 \\
$\mathrm{MgSO}_{4} \cdot 7 \mathrm{H}_{2} \mathrm{O}$ & 0,075 & $\mathrm{FeSO}_{4} \cdot 7 \mathrm{H}_{2} \mathrm{O} \mathrm{H}_{2} \mathrm{SO}_{4}$ & 0,0498 \\
$\mathrm{NaNO}_{3}$ & 0,25 & Metais * & 0,0541 \\
$\mathrm{~K}_{2} \mathrm{HPO}_{4}$ & 0,075 & $\mathrm{H}_{3} \mathrm{BO}_{3}$ & 0,115 \\
\hline
\end{tabular}

*As concentrações estão descritas na tabela para o meio BBM.

O estudo consistiu em três diferentes ensaios alterando a razão Carbono/Nitrogênio (20, 40 e 60), de modo a verificar o efeito de cada razão sobre o crescimento de biomassa e acúmulo de lipídeos.

As fermentações para cada relação $\mathrm{C} / \mathrm{N}$ (Tabela 2) foram realizadas em frascos erlenmeyer âmbar aletados de $250 \mathrm{~mL}$, com volume útil de $100 \mathrm{~mL}$, contendo solução de meio BBM, $10 \mathrm{~g} / \mathrm{L}$ de glicose anidra e $\mathrm{NaNO}_{3}$ de acordo com os valores da Tabela 2. $\mathrm{O} \mathrm{pH}$ foi corrigido para 6,8 e os experimentos foram realizados em duplicata.

Tabela 2 - Relação Carbono/Nitrogênio

\begin{tabular}{llll}
\hline Composição & Frascos & Relação $\mathrm{C} / \mathrm{N}$ & $\mathrm{NaNO}_{3}(\mathrm{~g} / \mathrm{L})$ \\
\hline 1 & 2 & 20 & 1,21 \\
2 & 2 & 40 & 0,6 \\
3 & 2 & 60 & 0,4 \\
\hline
\end{tabular}


O inóculo foi transferido em uma proporção de $10 \%$ (v/v) e foram incubados em shaker a $26^{\circ} \mathrm{C}$ por $96 \mathrm{~h}$, com agitação de $150 \mathrm{rpm}$. A cada $24 \mathrm{~h}$ foram retiradas amostras para análises de biomassa, glicose e nitrato. A concentração de biomassa foi determinada por densidade ótica, através da leitura espectrofotométrica a $680 \mathrm{~nm}$, utilizando água como solução de referência. A concentração de glicose foi determinada por análise colorimétrica com método glicose oxidase (kit enzimático Glicose Bioliquid, Laborclin) do sobrenadante previamente filtrado. Para a análise, $10 \mu \mathrm{L}$ da amostra foram adicionados a $1 \mathrm{~mL}$ do reagente enzimático e incubados a $37^{\circ} \mathrm{C}$ por $10 \mathrm{~min}$. Após resfriamento foi feita leitura de absorbância em espectrofotômetro a $505 \mathrm{~nm}$. A concentração de nitrato foi determinada por densidade ótica, segundo Ho et al. (2013). Para a análise, $0,5 \mathrm{~mL}$ do sobrenadante filtrado foi diluído com água deionizada e feito a leitura a $220 \mathrm{~nm}$ com cubeta de quartzo.

Após $96 \mathrm{~h}$, a biomassa foi centrifugada e armazenada a $-18^{\circ} \mathrm{C}$. As amostras congeladas foram liofilizadas para extração e quantificação de lipídios pelo método Ratzlaff. Esse método gravimétrico consistiu em pesar $0,5 \mathrm{~g}$ de biomassa liofilizada em tubos de ensaio, adicionar 10 $\mathrm{mL}$ de $\mathrm{HCl} 8 \mathrm{M}$ e deixar em banho-maria a $70^{\circ} \mathrm{C}$ por $10 \mathrm{~min}$. Em seguida, os tubos foram resfriado até temperatura ambiente e adicionado $10 \mathrm{~mL}$ de álcool etílico e $30 \mathrm{~mL}$ de éter de petróleo. Por fim, as amostras foram centrifugadas por $5 \mathrm{~min}$ a $2000 \mathrm{rpm}$ e a fase superior foi transferida para um balão de rotaevaporador, previamente tarado. Após o solvente ser evaporado, os balões foram levados a estufa à $60^{\circ} \mathrm{C}$ e 24 horas, ou até atingirem massa constante, resfriados a temperatura ambiente e pesados.

\section{RESULTADOS E DISCUSSÃO}

A partir do objetivo inicial de investigar o efeito de diferentes razões $\mathrm{C} / \mathrm{N}$ sobre o crescimento heterotrófico de microalgas bem como o acúmulo de lipídeos, obtiveram-se as Figuras 1, 2 e 3 que apresentam a variação com o tempo da concentração de glicose, nitrato e biomassa, para as razões C/N 20, 40 e 60, respectivamente.

Figura 1: Cinética de concentração de biomassa, glicose e nitrato residual para razão C/N 20 .

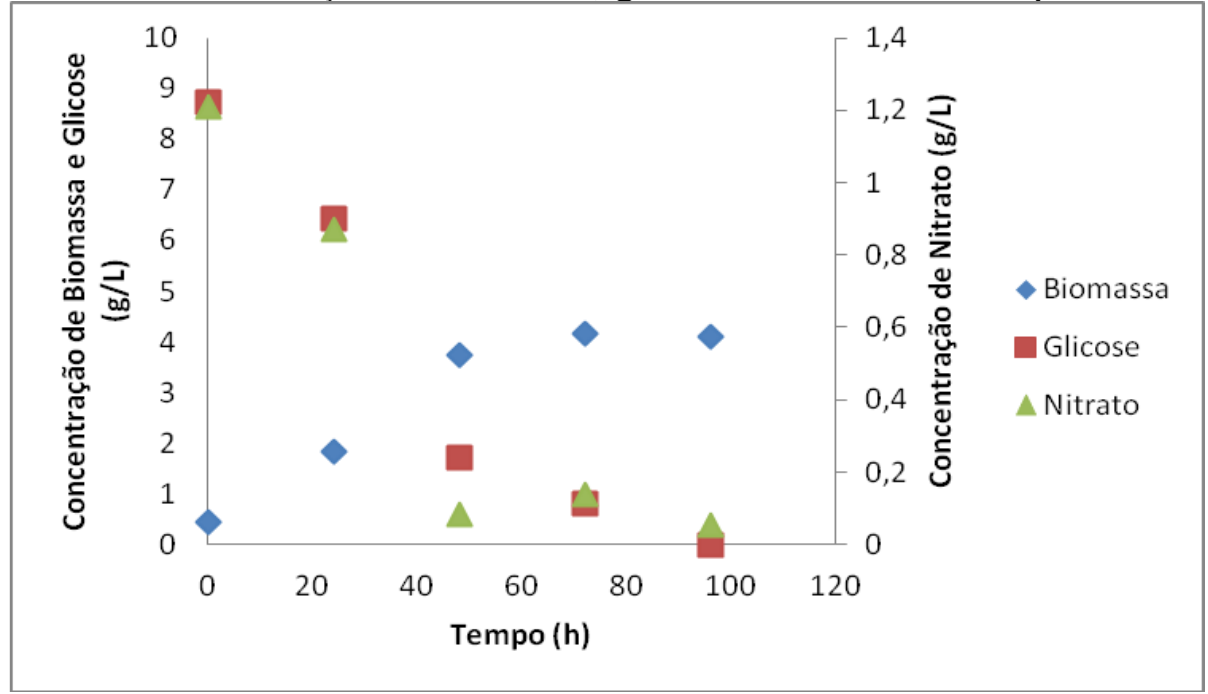


Figura 2: Cinética de concentração de biomassa, glicose e nitrato residual para razão C/N 40.

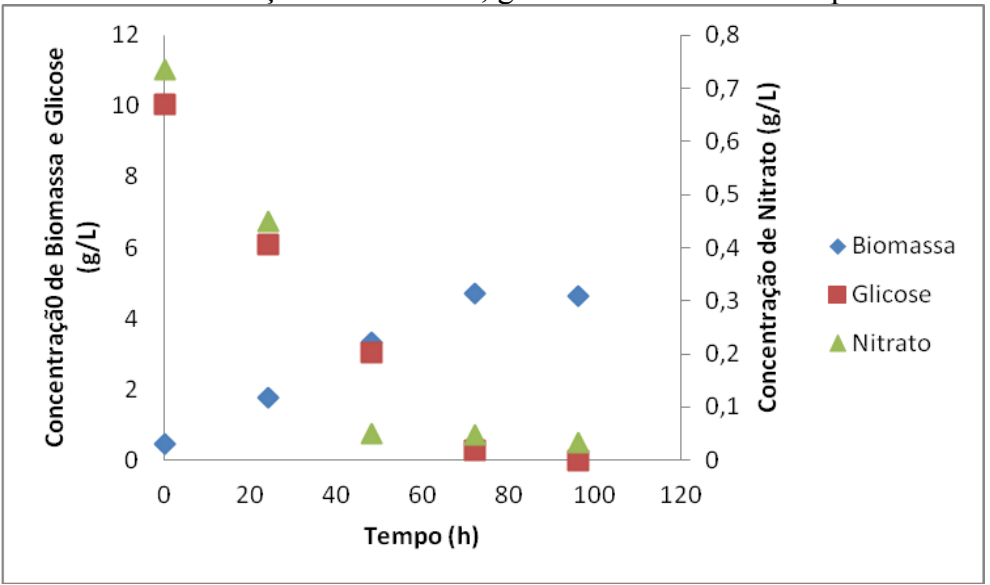

Figura 3: Cinética de concentração de biomassa, glicose e nitrato residual para razão C/N 60 .

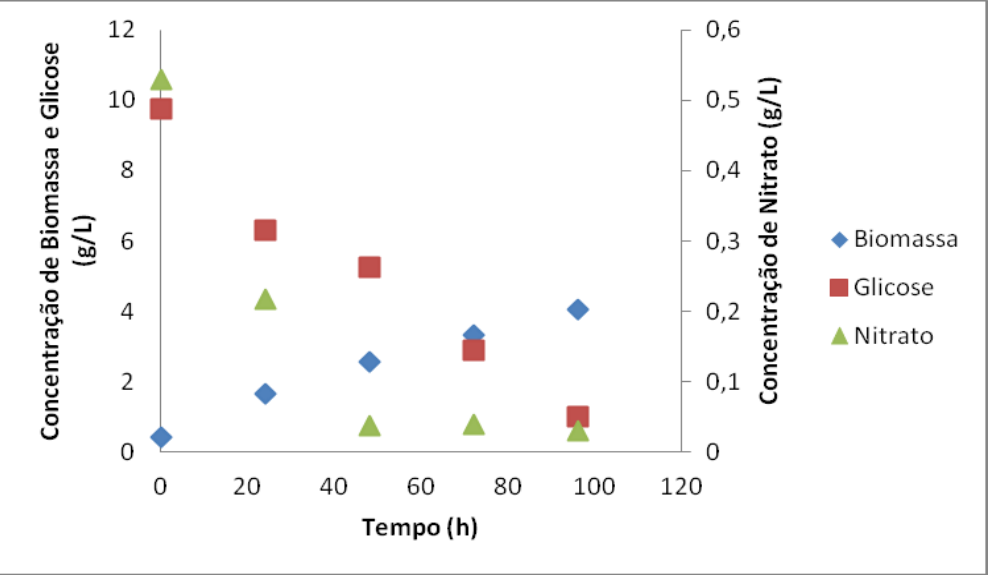

Os parâmetros cinéticos, tais como concentração inicial e máxima de biomassa velocidade específica máxima de crescimento, porcentagem de lipídeos e produtividade dé biomassa estão apresentados na Tabela 3 para cada condição de fermentação.

Tabela 3 - Parâmetros cinéticos de crescimento da C. vulgaris.

\begin{tabular}{cccccc}
\hline $\begin{array}{c}\text { Relação } \\
\mathrm{C} / \mathrm{N}\end{array}$ & $\begin{array}{c}\text { Concentração } \\
\text { de Biomassa } \\
\text { Inicial } \\
(\mathrm{g} / \mathrm{L})\end{array}$ & $\begin{array}{c}\text { Concentração } \\
\text { de Biomassa } \\
\text { Máxima } \\
(\mathrm{g} / \mathrm{L})\end{array}$ & $\begin{array}{c}\text { Velocidade } \\
\text { específica } \\
\text { máxima de } \\
\text { crescimento } \\
\left(\mathrm{h}^{-1}\right)\end{array}$ & $\begin{array}{c}\text { \% Lipídeos } \\
\text { Produtividade } \\
\text { biomasima de } \\
\left(\mathrm{g} \mathrm{L}^{-1} \mathrm{~h}^{-1}\right)\end{array}$ \\
\hline 20 & $0,44 \pm 0,03$ & $4,17 \pm 0,01$ & $0,045 \pm 0,001$ & $19,50 \pm 1,90$ & $0,038 \pm 0,002$ \\
40 & $0,46 \pm 0,02$ & $4,71 \pm 0,09$ & $0,041 \pm 0,001$ & $32,62 \pm 0,83$ & $0,043 \pm 0,001$ \\
60 & $0,44 \pm 0,01$ & $4,05 \pm 1,72$ & $0,037 \pm 0,006$ & $33,58 \pm 0,43$ & $0,038] \pm 0,013$ \\
\hline
\end{tabular}

Comentar [JL]: Arrumar a fonte e acrescentar erro. 
Na Tabela 3 é possível verificar que a maior concentração de biomassa ocorreu para a relação $\mathrm{C} / \mathrm{N} 40$, que foi de $(4,71 \pm 0,09) \mathrm{g} / \mathrm{L}$. Para as relações $\mathrm{C} / \mathrm{N} 20$ e 60 a concentração máxima de biomassa foi um pouco menor, $(4,17 \pm 0,01)$ e $(4,05 \pm 1,72) \mathrm{g} / \mathrm{L}$, respectivamente. As velocidades máximas de crescimento não tiveram grandes variações para as três condições estudadas. Para o acúmulo de lipídeos, nota-se que a maior porcentagem se deu para a relação $\mathrm{C} / \mathrm{N} 60(33,58 \pm 0,43 \%)$, enquanto que para a relação $\mathrm{C} / \mathrm{N} 20$ a porcentagem foi relativamente baixa $(19,50 \pm 1,90 \%)$. A produtividade máxima de biomassa, por sua vez, foi maior para a relação $\mathrm{C} / \mathrm{N} 40(0,043 \pm 0,001 \mathrm{~g} / \mathrm{L}$ h). Silaban et al. (2014) estudaram a modificação da relação $\mathrm{C} / \mathrm{N}$ para uma cultura conjunta de Chlorella vulgaris e Leptolyngbya sp. em heterotrofia e encontraram percentuais de lipídios bastante baixos comparados aos encontrados nesse trabalho, de $8,77 \%$ (base seca). Modificando a fonte de carbono, melhores resultados de percentual de lipídios foram alcançados.

\section{CONCLUS $\tilde{O} O$}

Os resultados obtidos neste trabalham permitem concluir que as fontes de carbono e nitrogênio, assim como as quantidades de cada uma no meio de cultivo e , portanto, a razão $\mathrm{C} / \mathrm{N}$ são de grande importância para alcançar melhores resultados de percentual de acúmulo de lipídeos e crescimento de biomassa.

\section{REFERÊNCIAS}

FRANCO, A. L. C., LOBO, I. P., DA CRUZ, R. S., TEIXEIRA, C. M. L. L., NETO, J. A. D. \& MENEZES, R. S. 2013. Biodiesel from Microalgae: Progress and Challenges. Quimica Nova, 36, 437-448.

GIORDANO, P. C., BECCARIA, A. J. \& GOICOECHEA, H. C. 2014. Rational design of a culture medium for the intensification of lipid storage in Chlorella sp Performance evaluation in air-lift bioreactor. Bioresource Technology, 158, 269-277.

GOUVEIA, L. 2011. Microalgae as a Feedstock for Biofuels, Springer.

HO, S. H., HUANG, S. W., CHEN, C. Y., HASUNUMA, T., KONDO, A. \& CHANG, J. S. 2013. Characterization and optimization of carbohydrate production from an indigenous microalga Chlorella vulgaris FSP-E. Bioresource Technology, 135, 157165.

KIRAN, B., KUMAR, R. \& DESHMUKH, D. Perspectives of microalgal biofuels as a renewable source of energy. Energy Conversion and Management.

LIU, J., CHEN, F. \& HUANG, J. 2011. Microalgae as Feedstocks for Biodiesel Production, INTECH Open Access Publisher.

RASHID, N., UR REHMAN, M. S., SADIQ, M., MAHMOOD, T. \& HAN, J.-I. 2014. Current status, issues and developments in microalgae derived biodiesel production. Renewable and Sustainable Energy Reviews, 40, 760-778.

SILABAN, A., BAI, R., GUTIERREZ-WING, M. T., NEGULESCU, I. I. \& RUSCH, K. A. 2014. Effect of organic carbon, $\mathrm{C}: \mathrm{N}$ ratio and light on the growth and lipid productivity of microalgae/cyanobacteria coculture. Engineering in Life Sciences, 14, 47-56.

SLADE, R. \& BAUEN, A. 2013. Micro-algae cultivation for biofuels: Cost, energy balance, environmental impacts and future prospects. Biomass \& Bioenergy, 53, 29-38.

WIJFFELS, R. H. \& BARBOSA, M. J. 2010. An Outlook on Microalgal Biofuels. Science, 329, 796-799. 
ZIOLKOWSKA, J. R. \& SIMON, L. 2014. Recent developments and prospects for algaebased fuels in the US. Renewable \& Sustainable Energy Reviews, 29, 847-853. 